\title{
Gut and Breast Milk Microbiota and Their Role in the Development of the Immune Function
}

\author{
Maria Elisabetta Baldassarre • Loredana Bellantuono • \\ Paola Mastromarino • Alfredo Miccheli • \\ Margherita Fanelli $\cdot$ Nicola Laforgia
}

Published online: 10 June 2014

(C) Springer Science + Business Media New York 2014

\begin{abstract}
The gastrointestinal immune system consists of both innate and adaptive immunity. The microbiota plays an important role in gastrointestinal immune function. In newborns, the maternal gut microbiota may either come across into the amniotic fluid or secrete factors that enter the amniotic fluid and affect the development of oral tolerance in utero. Adequate colonization must occur in the immediate postpartum period for the appropriate mucosal immune response since it is apparent that later attempts at colonization do not have the same impact. Human milk, being also a direct source of microbes, represents one of
\end{abstract}

M. E. Baldassarre $(\bowtie) \cdot$ L. Bellantuono $\cdot$ N. Laforgia Department of Biomedical Science and Oncology-Neonatology and NICU Section, Policlinico Hospital, University of Bari

"Aldo Moro", Piazza G.Cesare, 11, 70124 Bari, Italy

e-mail: mariaelisabetta.baldassarre@uniba.it

L. Bellantuono

e-mail: loredanabellantuono@gmail.com

N. Laforgia

e-mail: nicola.laforgia@uniba.it

P. Mastromarino

Department of Public Health and Infectious Diseases Section of Microbiology, University "Sapienza", P.le Aldo Moro, 5, 00185 Rome, Italy

e-mail: paola.mastromarino@uniroma1.it

\author{
A. Miccheli \\ Department of Chemistry, University "Sapienza", P.le Aldo \\ Moro, 5, 00185 Rome, Italy \\ e-mail: alfredo.miccheli@uniroma1.it \\ M. Fanelli \\ Department of Interdisciplinary Medicine, Policlinico Hospital, \\ University of Bari “Aldo Moro", Piazza G.Cesare, 11, \\ 70124 Bari, Italy \\ e-mail: margherita.fanelli@uniba.it
}

the main factors that play a critical role in influencing infant's microbiota composition.

Keywords Gut · Immune function · Gut microbiota . Breast milk microbiota $\cdot$ Newborns

\section{Introduction}

The gut is at the center of a complex system. Being the interface with the outside world, it is continuously exposed to microbial insults, so it must be capable of an efficient immune reaction to prevent adherence and colonization of potential pathogens. On the other hand, it must allow the passage of nutritional elements, growth factors, and maternal immunoglobulins (from breast milk) without reacting toward dietary and commensal microbial antigens, thus, achieving the immune tolerance.

\section{The Gut and its Role in Immune Function}

The gastrointestinal immune system consists of both innate and adaptive immunity. The first one, evolutionarily older, prompts an immediate but unspecific action against microorganisms through soluble factors (lactoferrin, peroxidase, lysozyme), phagocytic cells (macrophages and neutrophilic granulocytes), and pattern recognition receptors (PPRs) that, when they identify specific molecular patterns belonging to microorganisms (MAMPs), activate innate immune responses [1]

The main protagonist of the intestinal adaptive immunity is the "gut associated lymphoid tissue" (GALT). In the Peyer's Patch (PP) the M cells take up the antigens from the lumen and transport them in the lamina propria without 
degrading them, but releasing them intact to the antigenpresenting cells (APC's) processing. In the mesenteric lymph nodes the dendritic cells (DCs) present the antigens' peptides through class II major histocompatibility (MHC) molecules to $\mathrm{T}$ naïve cells, determining their phenotype: Th1 and Th2 subsets. Th1, through the secretion of IFN- $\gamma$, IL-2 and TNF- $\alpha$, modulate cell-mediated immunity against intracellular pathogens, whereas humoral immunity is orchestrated by Th2, via IL-4 and IL-6 [1].

When the microorganisms' (evolutionarily highly conserved) MAMPs bind the epithelial cells' PRRs, such as the Toll like receptors (TLRs), signaling cascades are activated, also via nuclear factor- $\mathrm{\kappa B}(\mathrm{NF}-\mathrm{\kappa B})$, and a prompt immune response through chemokine and others antimicrobial products is achieved [2••]. A harmful reaction against the commensal microorganisms that bind PRRs is avoided through complex mechanisms involving the IL-1 receptor associated kinase activation suppressor Tollinteracting protein and NF- $\mathrm{KB}$ agonist peroxisome proliferators-activated receptor $\gamma$ (PPAR- $\gamma$ ) [3].

Despite the several and efficient mechanisms that cooperate for the stratification of the microbiota, many commensal microorganisms make a breach in the gastrointestinal barrier and most of them are phagocytized by macrophages in the lamina propria. At this point the second important mechanism of the host-microbiota relationship comes into play: the compartmentalization of the gut flora, aiming at minimizing exposure of penetrant resident bacteria to the systemic immune system: these bacteria do not penetrate the systemic secondary lymphoid tissue $[2 \bullet \bullet]$.

The intestinal immune system is able to shape the composition of the microbiota through the release of antimicrobial peptides: $\alpha$-defensins by Paneth cells. It has been observed that mice with deficient functional or overexpressed human $\alpha$-defensins, showed, even if there are no change in the total number of microorganisms, " $\alpha$-defensine-dependent" modifications in the composition of intestinal microbial community $[2 \bullet \bullet, 3-6]$.

The ability of the intestinal immune system to shape the microbiota is also suggested by many experiments in which several immune deficiencies cause a dysbiosis that may predispose to disease (as demonstrated in mice that lack the transcriptor factor T-bet [7], in mice lacking the bacterial flagellin receptor TLR5 [8], in mice deficient for epithelial cell expression of the inflammasome component NLRP6 [9॰]).

\section{The Intestinal Microbiota}

The prosperous community of microorganisms that inhabit our gut engages a vivacious cross-talk with our immune system with reciprocal influences that are essential for the wellbeing of the individual.
The microbiota consists of $10^{14}$ microorganisms: predominantly bacteria, mainly strictly anaerobes, but also viruses, protozoa, archae, and fungi. The adult microbiota comprises over 1,000 species and more than 7,000 different strains. The most abundant phyla are Bacteroidetes and Firmicutes [10].

Several factors influence the composition of the microbiota: the type of delivery, the genetic heritage, the early diet (breast milk or formula feeding and, later, weaning), medications, infections and stress [11•]. Despite a significant interpersonal variation in the composition of the microbiota, the gut flora undergoes typical changes on its composition during the various stages of life. Modifications in the composition, or increase of pathogenic bacteria, can compromise the health state of the individual [10].

The microbiota is part of the complex bidirectional communication system between the Central Nervous System and the gut, what is called the "brain-gut-enteric microbiota axis." Through this bidirectional communication network, signals from the brain can modulate the motor, secretor, and sensitive functions of the gut and, vice versa, visceral information from the gastrointestinal tract can influence the brain function.

Indeed, it has been observed that surprisingly not only the brain may modify the microbiota (animal studies demonstrate that psychological stress alters the gut flora), but inversely the microbiota can modulate the brain's function and behavior [12]. New knowledge about this "bottom-up" pathway comes from the role of the Lactobacillus' ingestion in the regulation of emotional behavior and GABA receptor expression via vagus nerve in mice [13••].

Our understanding of the metabolic, structural, and protective function of intestinal flora has dramatically improved thanks to experiments on germ-free mice. Several morphological alterations were shown: enlarged cecum, reduced intestinal surface area, increased enterochromaffin cell area, smaller Peyer's Patches, and smaller villous thickness [10].

The microbiota can also be considered part of the gastrointestinal mucosal barrier. In fact, it plays an important role in gastrointestinal immune function firstly by competing metabolically in the gut's lumen with pathogens for nutrients and receptors. Furthermore, it is also responsible for the release of antimicrobial compounds and it avoids the adherence and colonization of the gut by others pathogens, also by preventing via cell signaling process the release of virulence factors. It also degrades luminal antigens: in germ-free animals there is an increased transport of antigens across the intestinal epithelium [1]. The suppression in vitro of lymphocyte proliferation and IL-4 production, by cow's milk casein hydrolyzed with enzymes derived from lactobacilli, supports the immunomodulatory role of the antigens degradation by microbiota $[14,15]$. 
In addition to its direct action against the pathogens, there is evidence demonstrating that some members of the commensal microbiota, such as E. coli, Bifidobacterium, Lactobacillus can interfere with some epithelial-apoptotic pathways triggered by other pathogens, thus increasing intestinal epithelial cell survival [16]. It has been shown also that the microbiota is involved in an increased epithelial cell proliferation and epithelium integrity, through translocation of the tight junction proteins and up regulation of genes involved in desmosome maintenance [17••].

The microbiota induces the repair of damaged intestinal epithelium through a MyD88-dependent process that can be rescued in microbe-depleted animals by gavage with bacterial lipopolysaccharides (LPS) [2••].

Germ-free mice lack isolated lymphoid follicles in the small intestine and also in IgAs and CD $8 \alpha \beta$ intraepithelial lymphocytes $[2 \cdot \bullet]$.

In addition to the induction of host innate responses, the microbiota exerts also a strong influence on shaping of $\mathrm{T}$ cells subsets. Both (the opposite) anti-inflammatory and proinflammatory role can be attributed to the commensal flora [18]. For example, mice colonized by a SFB-deficient microbiota lack mucosal Th17 cells, whereas colonization of mice with segmented filamentous bacteria (SFB) increases the share of Th17 cells, that absolve a proinflammatory function such as the Th1 [19, 20].

On the contrary, other components of the microbiota exert an anti-inflammatory function by inducing IL-10 expression or by polarizing the differentiation in $\mathrm{T}_{\text {reg }}$ cells. The capsular polysaccharide A (PSA) of the Bacteroides fragilis drives the differentiation of IL-10 secreting $\mathrm{T}_{\text {reg }}$ cells, whereas mutant Bacteroides fragilis lacking PSA shows a proinflammatory profile. However B. fragilis is responsible for a severe sepsis in immunocompromised hosts, demonstrating that the outcome of the interaction microbe-host does not depend only on the type of microbe involved in this process [18].

Captivating experiments on animals show the role of the microbiota on the systemic immunity, suggesting the implication of the dysbiosis in the genesis of extra-intestinal immune-mediated disease [2••]. In fact models for autoimmunity are dependent on colonization status: germfree mice have shown attenuation of disease in models of arthritis and experimental autoimmune encephalomyelitis (EAE); whereas, in models of Th17 cell-dependent arthritis and EAE, the monoassociation with SFB induces disease $[21,22 \cdot, 23]$.

Microbiota is also responsible for the activation of inflammation in immunocompromised hosts. Defective signaling through the phosphatase SHP-1 causes a microbiota-dependent autoinflammatory syndrome, that also takes place in mice lacking $\mathrm{B}$ and $\mathrm{T}$ cells. There are also a series of monogenic conditions of the nucleotide-binding oligomerization domain (NOD) receptor family considered to be autoinflammatory $[2 \bullet \bullet$.

Interesting studies suggest that the microbiota can play a protective role against autoimmune disease. In a study that used the non-obese diabetic mouse model, the incidence of type 1 diabetes was strictly related to the presence of pathogens and to the microbiota diversity [24, 25].

In allergy setting, a lower count of Lactobacillus and Bifidobacterium spp. in gut microbiota can precede the onset of the disease [26].A study showed an increased development of allergic disease in mice after oral antibiotic administration [27]. Another study compared two groups of suckling Sprague-Dawley rats, one received $100 \mathrm{mg} / \mathrm{kg} / \mathrm{d}$ Clamoxyl and the other one saline control. In the rats who received antibiotic, all bacteria were significantly reduced especially Lactobacillus, mainly in the colon. Affymetrix gene microarrays demonstrated that $10-30 \%$ of the genes undergoing maturational changes showed modulation by the antibiotic so that their normal pattern of maturation was either accelerated or slowed down. MHC genes, required for tolerization to luminal antigens, were markedly affected [28] However, studies investigating the protective role of pre and probiotic administration toward allergic disease are still conflicting [29-31].

\section{The Role of the Early Microbial Contact in Newborns}

In the development of the immune function, a pivotal role is played by the early microbial contact, that is likely to take place already in utero. That is why, in our understanding of the development of the immune development, the concept of "superorganism", already contemplating the symbiotic relationship between host and his microbiota, should be rethought in terms of the pair mother-fetus. In this "feto-maternal host microbe interaction" the first step of immunological education is represented by pre- and peri-partum time and the second one by lactation.

Several studies have refuted the old idea of a "sterile" fetus, after detecting microorganisms or their DNA in the placenta, in healthy membranes and amniotic fluid of term pregnancies. Because of the low levels of bacteria detected, it has been suggested that the aim of this "mother-to fetus efflux of commensal bacteria" is, rather than colonize, a way to educate the infant immune system to extrauterine life. Furthermore, although not yet fully understood, this microbial contact seems to be associated with a systemic

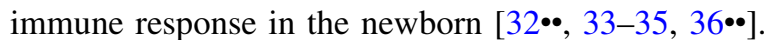

The maternal gastrointestinal tract seems to be the origin of this efflux of microorganisms toward the fetus. An elegant study on pregnant mice, after oral administration of a labeled Enterococcus faecium strain, showed a low level transfer of the labeled strain to the fetal gut and a higher level to the mammary glands $[36 \bullet \bullet]$. 
In addition to a direct intrauterine contact, there is also an indirect modulation of feto-placental immune responses during pregnancy. Maternal contact with farm animals and cats, that results in an higher maternal microbial contact during pregnancy, is able to influence the fetal immune system and, probably by altering the expression of TLR genes at birth, is responsible for a reduced risk of developing atopic dermatitis in the infant [37••]. A study on a mouse model of experimental allergic airway inflammation demonstrates that the maternal intranasal exposition to a bacterium isolated from cowsheds, the Acinetobacter lwoffii F78, suppresses TLRs in placental tissue, resulting in a lower risk for the progeny of developing asthma [38].

At birth, if the newborn passes through the birth canal, it picks up a health bolus of bacteria. These bacteria are stimulated to proliferate with the ingestion of oral feeding, particularly breast milk [39] and with weaning, resulting in adult-like microbiota that establishes itself usually about 1 year to 18 months of age. The large number of microorganisms communicate directly with epithelial cells, with DCs which penetrate between the epithelium and with underlying lymphoid elements [36••].

As a result of the interaction, mostly through tollreceptors, these lymphoid elements mature to produce an appropriate immune response which includes oral tolerance [40•].

Three main factors result in an inadequate initial colonization [41, 42]. One is birth by Cesarean section, which is a very common practice in developed countries. The second is the excessive use of perinatal antibiotics to mother during pregnancy or to the newborn in the perinatal period. Finally, premature delivery, where the infant may be born by cesarean section or may pass through the birth canal, but does so in such a rapid fashion that it does not pick up a large number of microorganisms, nor is its intestine able to respond to colonizing bacteria [43].

The intestinal microbiota of infants is very different from the one of adults and shows very important interindividual variability. The succession of bacterial species in the first months of life is very complex. Development of the intestinal microbiota in infants is characterized by rapid and large changes in microbial abundance, diversity, and composition.

Culture analysis of first meconium, collected within the first $2 \mathrm{~h}$ of life, demonstrated early colonization by facultative anaerobes of Firmicute phylum Enterococcus, Staphylococcus and other Enterobacteriaceae) [44]. In the first days of life other facultative anaerobes and strictly anaerobic bacteria such as Bifidobacterium, Lactobacillus, Clostridium, and Bacteroides colonize the gut of newborn. In infants of 1.5-5 months of age the most abundant classes of microorganisms present in the fecal samples are Bifidobacteriales $(80.6 \%)$, while second and third most abundant classes are Lactobacillales and Clostridiales (7.2 and $3.1 \%$, respectively) [45].

The correct sequence in the microbial species that colonize the intestine of the newborn in the first period of life leads to immune and metabolic programing that has long term influence on the risk of diseases. In fact the early microbial contact has been suggested to be determinant in the healthy immune maturation and development of tolerance; whereas an aberrant immune activation and responsiveness originated in this time may be the cause of allergic, metabolic, and autoimmune diseases in later life. Evidence demonstrates an association between cesarean section delivery and the risk of asthma, celiac disease, type 1 diabetes. Surprisingly, changes in the microbiota (less diverse, with lack of bifidobacterial species but increase in clostridium species) have been shown for each of these pathologies that are generally associated with an excessive or aberrant Th responsiveness [32••].

\section{Breast Feeding}

Far from being only a source of nutritional elements, the human milk represents the main stimulus to the development of the immune system for the vulnerable newborn infant. It provides passive immunoprotection through its antimicrobial factors, such as lactoferrin, lysozyme, defensins, peroxidase, IgAs, oligosaccharides, cathelicidin, TLRs with soluble CD14. Breast milk exerts also a strong immunomodulatory action and is a thriving source of colonizing bacteria [32••, 33].

Human milk represents one of the main factors that play a critical role in influencing infant's microbiota composition, also being a direct source of microbes. In fact, in aseptically collected breast milk culture-dependent methods have shown Staphylococcus, Streptococcus, Bifidobacterium species; whereas a core microbiome made up of 9 bacterial genera (Staphylococcus, Streptococcus, Serratia, Pseudomonas, Corynebacterium, Ralstonia, Propionibacterium, Sphingomonas, Bradyrhizobiaceae) emerges by pyrosequencing of $16 \mathrm{~S}$ rRNA genes in breast milk. Further complexity and inter-individual differences have been pointed out by other experiments based on cultivation and real-time qPCR [36••].

After lactose and lipids, the most abundant components (5-23 g/l) of human milk are oligosaccharides (HMOs), nondigestible glycans, that acts as prebiotics. Under the influence of several factors (lactation period, secretor status, maternal Lewis Blood Group, etc.) their production differs among women for sequence, charge and size of their molecular structure. This suggests the potential benefit of providing the infant with a different level of protection, depending on the specific growth stage, physiological state 
or on the environment. HMOs furthermore cooperate in improving absorption of minerals and in glucose homeostasis. Acting as prebiotics, HMOs stimulate changes, positive for the host, in the composition, in the activity and in the metabolism rate of the intestinal microflora, ensuring in this way a protective effect against pathogens. Some HMOs present similar glycan structural motifs that are supposed to act as decoy receptor for pathogens, preventing them from binding epithelial cells. HMOs improve the development of the mucosal immune system by facilitating the establishment of the microbiota, that finally results in an efficient prevention of disease. Moreover, the increase in the rate of Bifidobacteria that characterizes the breastfed microbiota has been attributed to oligosaccharides [36••].

\section{The Breast Milk Microbiota}

Several studies have pointed out a higher relative abundance of Bifidobacterium and Lactobacillus in the microbiota of breastfed infants [46-48] in comparison to formula-fed infants. It is interesting to note that these differences in the microbiota composition remain even after breast feeding is discontinued [32••]. Culture-dependent and -independent analysis of breast milk samples revealed the presence of Streptococcus and Staphylococcus genus, which correspond to early colonizers of the gut [49]. Bifidobacterium and Lactobacillus are also frequently detected, suggesting an important role of breast milk as a delivering system for beneficial bacteria [50] .

Other differences in formula-fed infants' microbiota have been shown: facultative anaerobes, Bacteroides, Clostridia, appear at higher level and frequency than in breastfed infants; predominant species within the Clostridia is the $C$. perfringens instead of $C$. difficile that is the most abundant Clostridium in breastfed infants [33]. Furthermore, breastfed, vaginally delivered term-infants showed a decrease in the microbiota rate of $C$. difficile and $E$. coli but an increase in beneficial bacteria, such as Bifidobacterium spp [42]. In fact, the microbial composition of breast milk is also likely dependent on the type of delivery. A different and less diverse community of microorganisms has been observed in milk samples from mothers who had elective cesarian section,

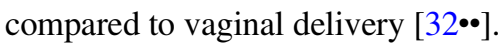

\section{Breast Feeding, Breast Milk Microbiota and Their Influence on the Immune Function}

With respect to the gut barrier function, formula-fed infants showed a higher epithelial permeability compared with breastfed infants, independently of gestational age at the birth. In animal models, formula feeding is associated with a higher incidence of bacterial translocation (that is the passage of bacteria from the gut to the mesenteric lymph nodes and other organs) with respect to breastfed newborns [33].

Several components of the human milk exert an immunomodulatory action. For example, soluble CD14, a receptor for lipopolysaccharide, regulates innate immune responses against Gram-negative bacteria. Furthermore, there is evidence of an inverse relationship between the breastmilk sCD14 and the risk of developing atopic disease [35].

Functional maternal immune cells, cytokines and cytokine receptor, growth factors, contained in human milk, modulate immune responses, and intestinal maturation in the infant. A pivotal role in this sense is played by TGF- $\beta$, very abundant in human milk. In fact the TGF- $\beta$ is involved in the breast milkinduced immune tolerance toward dietary antigens and indigenous intestinal microorganisms by induction of $\mathrm{T}_{\text {reg }}$ cells. TGF- $\beta$ restrains inflammatory responses in intestinal epithelial cells and $\mathrm{T}$ cells and exerts a modulation on the antigen-presenting cell. It stimulates the IgA production in the immunologically naïve neonate, thus improving the intestinal barrier function [35]. There is also evidence that TGF- $\beta$ influences the immune maturation in the immature human gut [32••].

A fascinating theory proposed by Rautava et al. suggests that: "breast milk might function as a vehicle to introduce maternal gut microbiota to the infant in tolerogenic immune milieu". Important data have contributed to this theory, for example, the observation of a reduced risk of atopic eczema in breastfed infants after maternal consumption of the probiotic L. rhamnosus GG, that determined a higher concentration of TGF- $\beta$ in breast milk. In line with this observation, a modulation of the responsiveness to TGF- $\beta$ in preterm proved possible after administration of the probiotic Bifidobacterium breve

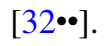

In animals, it has been demonstrated that during pregnancy and lactation there is an increased bacterial translocation and bacterially loaded DCs can easily reach the mammary gland via lymphatics and peripheral blood. Furthermore, bacteria and bacterial nucleic acids have also been detected in cells of human breast milk and in maternal peripheral blood mononuclear cells. Another elegant study has compared the flora of maternal feces, breast milk, and infant feces, highlighting an overlapping in number and type of bifidobacteria, whereas breast milk and infant feces present the same strains of staphylococci, bifidobacteria, and lactobacilli [32••].

\section{Modulation of Microbiota in Perinatal Period}

The increased incidence of allergic and autoimmune diseases, including Crohn's disease, type 1 diabetes, multiple 
Fig. 1 TGF- $\beta$ values in colostrum (T0) and mature milk (T30) in mothers who assumed VSL\#3 (VSL\#3) and in control mothers (CTR)

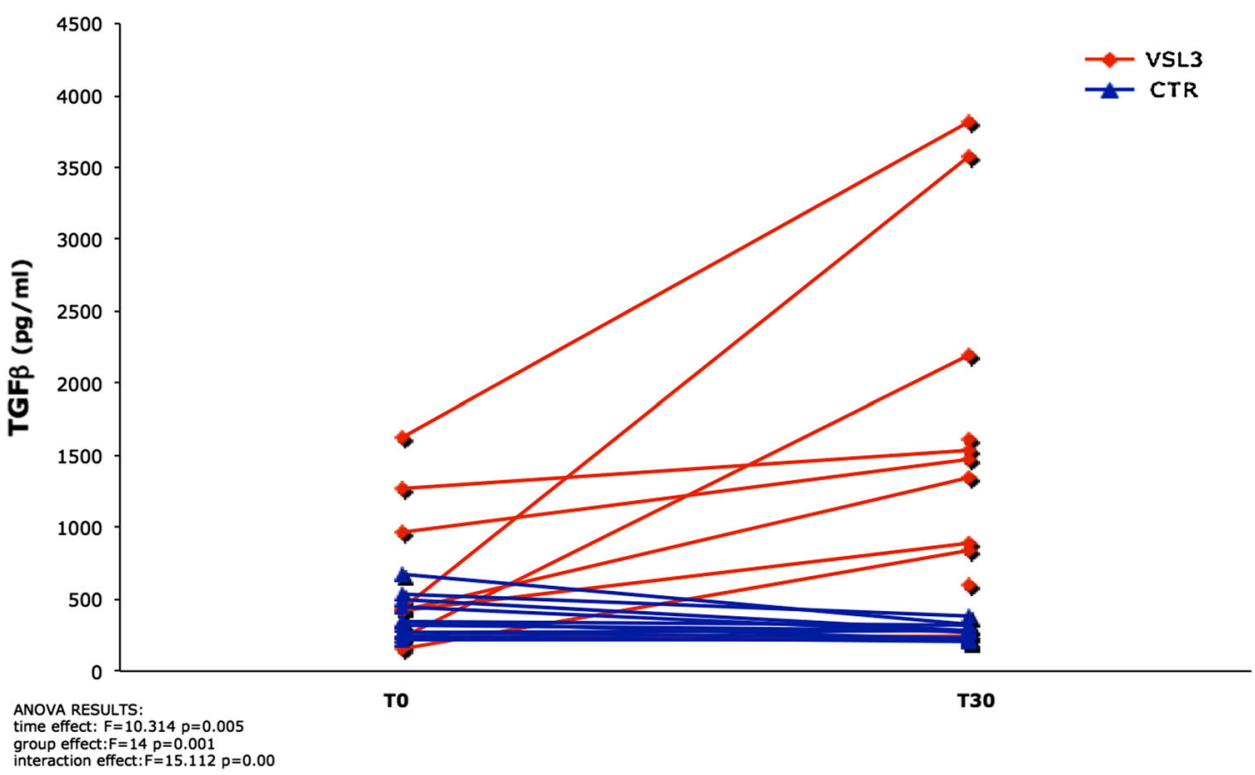

sclerosis, and celiac disease is mainly observed in children who are born with inadequate initial bacterial colonization. The theory is that adequate colonization must occur in the immediate postpartum period for the appropriate mucosal immune response to occur. In the absence of adequate colonization, despite attempts to upgrade this later in life, apparently does not have the same impact as colonization in the neonatal period.

The ability to modulate the development and the composition of the intestinal microbiota in order to improve health of both mother and child is the basic rationale for the use of probiotics. The administration of probiotics to the mother in the perinatal period results in changes of intestinal microbiota of women that are reflected in changes of immune factors and in microbiota of the newborn. The consumption of Lactobacillus GG by Finnish mothers before birth and during lactation induced modulation of colonization and growth of Bifidobacteria in the gut of their children, in particular by increasing the diversity of species of Bifidobacteria [51]. In addition, the administration to mothers during the last weeks of pregnancy of probiotics containing a strain of lactobacilli resulted in increased amount of B. longum fecal in infants [52] and has proven effective in protecting the child from atopic dermatitis [53]. The consumption of VSL\#3, a mixture of lactobacilli and bifidobacteria in the last month of pregnancy and during the first month of lactation resulted in a significant increase in breast milk concentrations of secretory IgA and antiinflammatory and immunomodulatory cytokines such as TGF- $\beta$ (Fig. 1) and IL-6 (Fig. 2) [54]. Furthermore, the concentration of lactobacilli and bifidobacteria in the intestinal microbiota of infants breastfed by mothers who took the VSL\#3 was higher compared with infants of control mothers [54]. A recent study [55] showed that oral intake of VSL\#3 in the last trimester of pregnancy is associated with a modulation of the vaginal microbiota and secretion of cytokines, with significantly higher levels of IL-4 and IL-6, with an anti-inflammatory effect, and reduction of Eotaxin, a proinflammatory chemokine. VSL\#3 intake was associated with a stabilization of vaginal immune responses and a global anti-inflammatory effect on the vaginal community. These changes in vaginal microbiota could have important implications in the prevention of vaginal infections and, therefore, of preterm birth.

\section{Conclusions}

The first contact with the bacteria during and immediately after birth has an important role in the development of the intestinal flora and then on the development of mucosal immune system of the newborn. Intestinal microbiota of the newborn is related to his susceptibility to infection and sensibility to antigens in the environment in the early years of life. The correct sequence in the microbial species that colonize the intestine of the newborn in the first period of life leads to immune and metabolic programing that has a long term influence on the risk of diseases.

Breast feeding is considered today the "second step" of immunological education. Far from being only a source of nutritional elements, the human milk represents for the vulnerable newborn infant the main stimulus to the development of his immune system. Our understanding of the interactions between microorganisms and the host is still in its infancy. We know very little of how different members of the indigenous microbiota influence each 
other. Furthermore, the notion of host-microbe interaction entails active communication on both the part of the host and the bacteria. One important focus area for future research is characterization of the activity of microbes and how it affects host physiology and vice versa. In order to assess the full impact of perinatal probiotic interventions, longer-term follow-up data from already conducted trials are necessary. New clinical trials with clearly defined target populations and clinical as well as immunologic, metabolic, and microbiological endpoints may be conducted and, eventually, recommendations for clinical use to improve patient care may hopefully be devised.

Disclosure Maria Elisabetta Baldassarre, Loredana Bellantuono, Paola Mastromarino, Alfredo Miccheli, Margherita Fanelli and Nicola Laforgia declare that they have no conflict of interest.

Human and Animal Rights and Informed Consent This article does not contain any studies with human or animal subjects performed by any of the authors.

\section{References}

Papers of particular interest, published recently, have been, highlighted as:

- Of importance,

-• Of major importance

1. Rautava S, Walker WA. Immunophysiology and nutrition of the gut. Nutrition in Pediatrics. 4th ed. 2008.

2. - Hooper LV, Littman DR, Macpherson AJ. Interactions between the microbiota and the immune system. Science 2012;336:1268-73. Advances in understanding of the interactions between resident microbes and the immune system and the implications of these findings for human health.

3. Salzman NH, Ghosh D, Huttner KM, Paterson Y, Bevins CL. Protection against enteric salmonellosis in transgenic mice expressing a human intestinal defensin. Nature. 2003;422:522-6.

4. Diebel LN, Liberati DM, Baylor AE III, et al. The pivotal role of tumor necrosis factor-alpha in signaling apoptosis in intestinal epithelial cells under shock conditions. J Trauma. 2005;58: 995-1001.

5. Planchon SM, Martins CA, Guerrant RL, Roche JK. Regulation of intestinal epithelial barrier function by TGF-beta 1 . Evidence for its role in abrogating the effect of a $\mathrm{T}$ cell cytokine. J Immunol. 1994;153:5730-9.

6. Otte JM, Cario E, Podolsky DK. Mechanisms of cross hyporesponsiveness to Toll-like receptor bacterial ligands in intestinal epithelial cells. Gastroenterology. 2004;126:1054-70.

7. Garrett WS, Lord GM, Punit S, Lugo-Villarino G, Mazmanian SK, Ito S, Glickman JN, Glimcher LH. Communicable ulcerative colitis induced by T-bet deficiency in the innate immune system. Cell. 2007;131:33-45.

8. Vijay-Kumar M, Aitken JD, Carvalho FA, Cullender TC, Mwangi S, Srinivasan S, Sitaraman SV, Knight R, Ley RE, Gewirtz AT. Metabolic syndrome and altered gut microbiota in mice lacking Toll-like receptor 5. Science. 2010;328:228-31.
9. • Elinav E, Strowig T, Kau AL, Henao-Mejia J, Thaiss CA, Booth CJ, Peaper DR, Bertin J, Eisenbarth SC, Gordon JI, Flavell RA. NLRP6 inflammasome regulates colonic microbial ecology and risk for colitis. Cell. 2011;145:745-57. The importance of the gut microbiota in the pathogenesis of various autoimmune disorders that manifest outside of the gastrointestinal tract.

10. Grenham S, Clarke G, Cryan JF, Dinan TG. Brain-gut-microbe communication in health and disease. Front Physiol. 2011;2:94.

11. - Bercik P, Collins SM, Verdu EF. Microbes and the gut-brain axis. Neurogastroenterol Motil. 2012;24:405-13. The current evidence for a role of bacteria (commensals, probiotics, and pathogens) as key modulators of gut-brain communication.

12. Collins SM, Bercik P. The relationship between intestinal microbiota and the central nervous system in normal gastrointestinal function and disease. Gastroenterology. 2009;136:2003-14.

13. $\bullet$ Bravo JA, Forsythe P, Chew MV, Escaravage E, Savignac HM, Dinan TG, Bienenstock J, Cryan JF. Ingestion of Lactobacillus strain regulates emotional behavior and central GABA receptor expression in a mouse via the vagus nerve. Proc Natl Acad Sci U S A. 2011;108:16050-5. The important role of bacteria in the bidirectional communication of the gut-brain axis. Certain organisms may prove to be useful therapeutic adjuncts in stressrelated disorders such as anxiety and depression.

14. Sütas Y, Hurme M, Isolauri E. Down-regulation of anti-CD3 antibody-induced IL-4 production by bovine caseins hydrolysed with Lactobacillus GG-derived enzymes. Scand J Immunol. 1996;43:687-9.

15. Sütas Y, Soppi E, Korhonen H, Syväoja EL, Saxelin M, Rokka T, Isolauri E. Suppression of lymphocyte proliferation in vitro by bovine caseins hydrolyzed with Lactobacillus casei GG-derived enzymes. J Allergy Clin Immunol. 1996;98:216-24.

16. Ohland CL, Macnaughton WK. Probiotic bacteria and intestinal epithelial barrier function. Am J Physiol Gastrointest Liver Physiol. 2010;298(6):G807-19.

17. •- Ashida H, Ogawa M, Kim M, Mimuro H, Sasakawa C. Bacteria and host interactions in the gut epithelial barrier. Nat Chem Biol. 2011;8:36-45. An overview of the components of the mucosal barrier and the bacterial stratagems that circumvent these barriers with particular emphasis on the roles of bacterial effector proteins.

18. Cerf-Bensussan N, Gaboriau-Routhiau V. The immune system and the gut microbiota: friends or foes? Nat Rev Immunol. 2010;10:735-44.

19. Gaboriau-Routhiau V, Rakotobe S, Lécuyer E, Mulder I, Lan A, Bridonneau C, Rochet V, Pisi A, De Paepe M, Brandi G, Eberl G, Snel J, Kelly D, Cerf-Bensussan N. The key role of segmented filamentous bacteria in the coordinated maturation of gut helper $\mathrm{T}$ cell responses. Immunity. 2009;31:677-89.

20. Ivanov II, Atarashi K, Manel N, Brodie EL, Shima T, Karaoz U, Wei D, Goldfarb KC, Santee CA, Lynch SV, Tanoue T, Imaoka A, Itoh K, Takeda K, Umesaki Y, Honda K, Littman DR. Induction of intestinal Th17 cells by segmented filamentous bacteria. Cell. 2009;139:485-98.

21. Abraham C, Cho JH. IL-23 and autoimmunity: new insights into the pathogenesis of inflammatory bowel disease. Annu Rev Med. 2009;60:97-110.

22. - Lee YK, Menezes JS, Umesaki Y, Mazmanian SK. Proinflammatory T-cell responses to gut microbiota promote experimental autoimmune encephalomyelitis. Proc Natl Acad Sci U S A. 2011;108 Suppl 1:4615-22. The intestinal microbiota profoundly impacts the balance between pro- and antiinflammatory immune responses during experimental autoimmune encephalomyelitis and suggest that modulation of gut bacteria may provide therapeutic targets for extraintestinal inflammatory diseases such as Multiple Sclerosis. 
23. Wu HJ, Ivanov II, Darce J, Hattori K, Shima T, Umesaki Y, Littman DR, Benoist C, Mathis D. Gut-residing segmented filamentous bacteria drive autoimmune arthritis via T helper 17 cells. Immunity. 2010;32:815-27.

24. Ohsugi T, Kurosawa T. Increased incidence of diabetes mellitus in specific pathogen-eliminated offspring produced by embryo transfer in NOD mice with low incidence of the disease. Lab Anim Sci. 1994;44:386-8.

25. Leiter E. The role of microorganisms in non-infectious disease, R. d. Vries, I. Cohen, and J. J. v. Rood, eds. Berlin: Springer Verlag. 1990;39-55.

26. Sjögren YM, Jenmalm MC, Böttcher MF, Björkstén B, Sverremark-Ekström E. Altered early infant gut microbiota in children developing allergy up to 5 years of age. Clin Exp Allergy. 2009;39:518-26.

27. Noverr MC, Falkowski NR, McDonald RA, McKenzie AN, Huffnagle GB. Development of allergic airway disease in mice following antibiotic therapy and fungal microbiota increase: role of host genetics, antigen, and interleukin-13. Infect Immun. 2005;73:30-8.

28. Schumann Al, Nutten S, Donnicola D, Comelli EM, Mansourian R, Cherbut C, Corthesy-Theulaz I, Garcia-Rodenas C. Neonatal antibiotic treatment alters gastrointestinal tract developmental gene expression and intestinal barrier transcriptome. Physiol Genomics. 2005;23:235-45.

29. Kopp MV, Goldstein M, Dietschek A, Sofke J, Heinzmann A, Urbanek R. Lactobacillus GG has in vitro effects on enhanced interleukin-10 and interferon-gamma release of mononuclear cells but no in vivo ef- fects in supplemented mothers and their neonates. Clin Exp Allergy. 2008;38:602-10.

30. Abrahamsson TR, Jakobsson T, Böttcher MF, et al. Probiotics in prevention of IgE-associated eczema: a double-blind, randomized, placebo-controlled trial. J Allergy Clin Immunol. 2007;119: 1174-80.

31. Wickens K, Black PN, Stanley TV. Probiotic study group. A differential effect of 2 probiotics in the prevention of eczema and atopy: a double-blind, randomized, placebo-controlled trial. J Allergy Clin Immunol. 2008;122:788-94.

32. • Rautava S, Luoto R, Salminen S, Isolauri E. Microbial contact during pregnancy, intestinal colonization and human disease. Nat Rev Gastroenterol Hepatol. 2012;9:565-76. The authors describe the current body of knowledge regarding perinatal microbial contact, initial intestinal colonization and its association with human disease, as well as means of modulating early hostmicrobe interaction to reduce the risk of disease in the child.

33. Le Huërou-Luron I, Blat S, Boudry G. Breast- vs formula-feeding: impacts on the digestive tract and immediate and long-term health effects. Nutr Res Rev. 2010;23:23-36.

34. Newburg DS, Walker WA. Protection of the neonate by the innate immune system of developing gut and of human milk. Pediatr Res. 2007;61:2-8.

35. Rautava S, Walker WA. Academy of Breastfeeding Medicine founder's lecture 2008: breastfeeding-an extrauterine link between mother and child. Breastfeed Med. 2009;4:3-10.

36. •• Thum C, Cookson AL, Otter DE, McNabb WC, Hodgkinson AJ, Dyer J, Roy NC. Can nutritional modulation of maternal intestinal microbiota influence the development of the infant gastrointestinal tract? J Nutr. 2012;142:1921-8. This review examines the manipulation of the maternal GIT microbiota composition through the use of probiotics and/or prebiotics and subsequent consequences for the health of the offspring.

37. • Roduit C, Wohlgensinger J, Frei R, Bitter S, Bieli C, Loeliger S, Büchele G, Riedler J, Dalphin JC, Remes S, Roponen M, Pekkanen J, Kabesch M, Schaub B, von Mutius E, Braun-Fahrländer C, Lauener R; PASTURE Study Group. Prenatal animal contact and gene expression of innate immunity receptors at birth are associated with atopic dermatitis. J Allergy Clin Immunol. 2011;127:179-85, 185.e1. An innovative epidemiologic study that have indicated that maternal contact with farm animals, which presumably entails increased microbial contact during pregnancy, modulates fetal innate immune gene expression and protects the infants from development of dermatitis.

38. Conrad ML, Ferstl R, Teich R, Brand S, Blümer N, Yildirim AO, Patrascan CC, Hanuszkiewicz A, Akira S, Wagner H, Holst O, von Mutius E, Pfefferle PI, Kirschning CJ, Garn H, Renz H. Maternal TLR signaling is required for prenatal asthma protection by the nonpathogenic microbe Acinetobacter lwoffii F78. J Exp Med. 2009;206:2869-77.

39. Rautava S, Lu L, Nanthakumar NN, Dubert-Ferrandon A, Walker WA. TGF- $\beta 2$ induces maturation of immature human intestinal epithelial cells and inhibits inflammatory cytokine responses induced via the NF- $\kappa \mathrm{B}$ pathway. J Pediatr Gastroenterol Nutr. 2012;54:630-8.

40. - Kaplan JL, Shi HN, Walker WA. The role of microbes in developmental immunologic programming. Pediatr Res 2011; 69:465-72. Exposure to microbes before conception, during gestation, and in the neonatal period have profound effects on the developing immune system.

41. Penders J, Thijs C, Vink C, Stelma FF, Snijders B, Kummeling I, van den Brandt PA, Stobberingh E. Factors influencing the composition of the intestinal microbiota in early infancy. Pediatrics. 2006;118:511-21.

42. Adlerberth I, Wold AE. Establishment of the gut microbiota in Western infants. Acta Paediatr. 2009;98:229-38.

43. Nanthakumar N, Meng D, Goldstein AM, Zhu W, Lu L, Uauy R, Llanos A, Claud EC, Walker WA. The mechanism of excessive intestinal inflammation in necrotizing enterocolitis: an immature innate immune response. PLoS ONE. 2011;6:e17776.

44. Jiménez E, Marín ML, Martín R, Odriozola JM, Olivares M, Xaus J, Fernández L, Rodríguez JME, et al. Is meconium from healthy newborns actually sterile? Res Microbiol. 2008;159: 187-93.

45. Turroni F, Peano C, Pass DA, Foroni E, Severgnini M, Claesson MJ, Kerr C, Hourihane J, Murray D, Fuligni F, Gueimonde M, Margolles A, De Bellis G, O'Toole PW, van Sinderen D, Marchesi JR, Ventura M. Diversity of bifidobacteria within the infant gut microbiota. PLoS One. 2012;7:e36957.

46. Palmer C, Bik EM, DiGiulio DB, Relman DA, Brown PO. Development of the human infant intestinal microbiota. PLoS Biol. 2007;5:e177.

47. Harmsen HJ, Wildeboer-Veloo AC, Raangs GC, Wagendorp AA, Klijn N, Bindels JG, Welling GW. Analysis of intestinal flora development in breast-fed and formula-fed infants by using molecular identification and detection methods. J Pediatr Gastroenterol Nutr. 2000;30:61-7.

48. Knol J, Scholtens P, Kafka C, Steenbakkers J, Gro S, Helm K, Klarczyk M, Schöpfer H, Böckler HM, Wells J. Colon microflora in infants fed formula with galacto- and fructo-oligosaccharides: more like breast-fed infants. J Pediatr Gastroenterol Nutr. 2005;40:36-42.

49. Collado MC, Delgado S, Maldonado A, Rodríguez JM. Assessment of the bacterial diversity of breast milk of healthy women by quantitative real-time PCR. Lett Appl Microbiol. 2009;48: 523-8.

50. Fernandez L, Langa S, Martín V, Maldonado A, Jiménez E, Martín R, Juan M, Rodríguez JM. The human milk microbiota: origin and potential roles in health and disease. Pharmacol Res. 2013;69:1-10.

51. Gueimonde M, Sakata S, Kalliomäki M, Isolauri E, Benno Y, Salminen S. Effect of maternal consumption of Lactobacillus GG on transfer and establishment of fecal bifidobacterial microbiota in neonates. J Pediatr Gastroenterol Nutr. 2006;42:166-70. 
52. Lahtinen SJ, Boyle RJ, Kivivuori S, Oppedisano F, Smith KR, Robins-Browne R, Salminen SJ, Tang ML. Prenatal probiotic administration can influence Bifidobacterium microbiota development in infants at high risk of allergy. J Allergy Clin Immunol. 2009;123:499-501.

53. Rautava S, Kainonen E, Salminen S, Isolauri E. Maternal probiotic supplementation during pregnancy and breast-feeding reduces the risk of eczema in the infant. J Allergy Clin Immunol. 2012;130:1355-60.
54. Baldassarre ME, Cacciotti F, Miccheli A, Urbano F, Laforgia N, Mastromarino P. VSL\#3 probiotic maternal supplementation affects breast milk composition and newborn faeces microbiota. Dig Liver Dis. 2012;44:S253.

55. Vitali B, Cruciani F. Baldassarre ME, Capursi T, Spisni E, Maria Valerii MC, Candela M, Turroni S and Brigidi P. Dietary supplementation with probiotics during late pregnancy: outcome on vaginal microbiota and cytokine secretion. BMC Microbiol. 2012;12:236-40. 